\title{
Anterior instrumented fusion for adolescent idiopathic scoliosis
}

\author{
Michael Ruf ${ }^{1}, J_{o ̈ r g}$ Drumm $^{1}$, Dezsö Jeszenszky ${ }^{2}$ \\ ${ }^{1}$ Center for Spinal Surgery, Orthopedics, and Traumatology, SRH Klinikum Karlsbad-Langensteinbach, Karlsbad, Germany; ${ }^{2}$ Spinal Surgery, \\ Orthopedics and Neurosurgery, Schulthess Klinik, Zürich, Switzerland \\ Contributions: (I) Conception and design: All authors; (II) Administrative support: All authors; (III) Provision of study materials or patients: All \\ authors; (IV) Collection and assembly of data: All authors; (V) Data analysis and interpretation: All authors; (VI) Manuscript writing: All authors; (VII) \\ Final approval of manuscript: All authors. \\ Correspondence to: Michael Ruf. SRH-Klinikum Karlsbad-Langensteinbach, Guttmannstrasse 1, D-76307 Karlsbad, Germany. Email: Michael.ruf@srh.de.
}

\begin{abstract}
Adolescent idiopathic scoliosis (AIS) is a complex three-dimensional deformity of the spine consisting of a lateral curvature, apical vertebral rotation, and an impairment of the sagittal profile. Surgical options include anterior and posterior approaches. Anterior instrumented fusion is suitable in Lenke type 1 and 5 curves. It supplies excellent results in coronal plane correction and is superior in the restoration of the sagittal profile and apical derotation. Fusion is shorter compared to posterior correction, and the complication rate is low. Pulmonary function is impaired postoperatively but recovers within a few years.
\end{abstract}

Keywords: Adolescent idiopathic scoliosis (AIS); anterior approach; correction; derotation; thoracic kyphosis; pulmonary function

Submitted Sep 24, 2019. Accepted for publication Oct 28, 2019.

doi: 10.21037/atm.2019.11.84

View this article at: http://dx.doi.org/10.21037/atm.2019.11.84

\section{Introduction}

Alan Dwyer introduced anterior correction of adolescent idiopathic scoliosis (AIS) in the early seventies (1). He used an anterior cable and screw instrumentation system to correct and stabilize scoliotic deformities. The technique showed good results in the frontal plane; however, due to the anterior compression, a tendency to kyphosis was inherent in this technique. This was favorable in the thoracic region but disastrous in the lumbar spine (2). Besides, the pseudarthrosis rate was high.

The anterior technique was further developed by Klaus Zielke (3). He introduced a flexible solid threaded rod/screw system with the possibility of derotation with a special tool (derotator). In the following, the fusion rates improved; however, the problem of kyphosis in the lumbar spine remained.

Subsequent developments improved the results of anterior scoliosis surgery. The introduction of screw/rod systems with stiff rods (single and double rods) allowed for a new correction maneuver: a correction was achieved by rotation of a pre-bent stiff rod. The problem of kyphosis in the lumbar spine was overcome by the use of structural grafts/ cages in the disc space. By a combination of these techniques (rod rotation, anterior support, and compression via the instrumentation), the complete correction of the deformity in all three planes was achieved with the high stability of the construct. The pseudarthrosis rate decreased (4-7).

Anterior correction of AIS was the predominant procedure at the end of the last century, but numbers decreased markedly in favor of the posterior procedures. However, in the same period, the number of fused levels increased considerably (e.g., two added segments in Lenke 1 curves) (8). Long-term follow-up studies are imperative to evaluate these effects in the course of the patient's lifetime.

\section{Indication}

Anterior correction in AIS should be considered in single thoracic and lumbar deformities with flexible compensatory curves. These are type 1 and type 5 curvatures according to Lenke's classification.

The indication for anterior correction and fusion depends on the degree of scoliosis in the frontal plane, the amount of kyphosis/lordosis in the sagittal plane, the age 


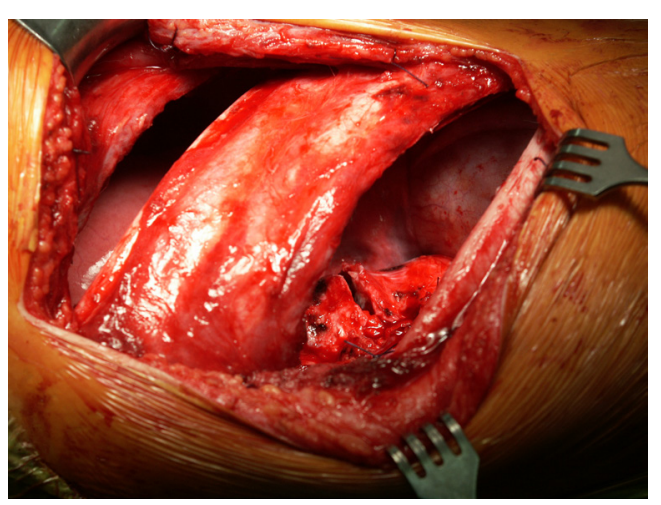

Figure 1 Surgical approach to the thoracic spine via a double thoracotomy.

of the patient, the expected further progression, and the cosmetic impairment (rib hump).

In the thoracic spine, the indication for surgery is starting with a Cobb angle of 40 to 50 degrees. The ideal patient for anterior instrumented fusion is the single thoracic curve (Lenke type $1 \mathrm{~A}-\mathrm{C}$ ) with pronounced hypokyphosis (T5-12 sagittal modifier-or N). Since the anterior correction includes a shortening of the anterior column of the spine and is therefore kyphogenic, it should not be applied in primarily hyperkyphotic cases (rare), or in very young children that tend to develop hyperkyphosis during further growth due to the anterior tether.

In the lumbar spine, indication for surgical correction is given with an angle of 35 to 40 degrees. Lenke type 5 curves are ideal for anterior instrumented fusion, since the length of fusion is shorter than in posterior correction and derotation is more effective.

\section{Diagnostics}

Radiographs of the whole spine in AP and lateral view, as well as bending films, are inevitable to assess the extent, localization, and flexibility of the main curve as well as the compensatory curves. MRI of the whole spine is recommended to exclude intraspinal pathologies. In severe cases, a low-dose CT and traction films should be considered.

The deformity is then classified according to Lenke (9).

\section{Technique}

\section{Thoracic curves (Lenke type 1)}

In the thoracic spine, correction, and fusion are performed from end vertebra to end vertebra. For typical Lenke 1 curves, the uppermost instrumented vertebra is T5 or T6, the lowest T11, T12, or L1. The patient is placed on a hinged table (hinge at the apex of the curve) in the lateral position with the convexity of the curve up. The approach is performed via a single skin incision and a double thoracotomy, the upper one between $4^{\text {th }}$ and $5^{\text {th }}$ respectively between $5^{\text {th }}$ and $6^{\text {th }}$ rib with distal osteotomy of the rib, the lower one usually 4 rib spaces lower. This allows for access to the disc spaces in the trajectory of the discs with an unobstructed view of the posterior ligaments. The rib heads overlapping the discs are removed. Depending on the severity of the rib hump, the proximal parts of the ribs are resected. The discs are removed completely, including the contralateral and posterior annulus, to achieve sufficient segmental mobility for correction. The longitudinal fibers of the longitudinal posterior ligament are visible and should be partially disrupted by distraction in very rigid cases. Removal of the discs results in a shortening of the anterior column of the spine, thus restoring a physiological thoracic kyphosis. In severe hypokyphosis the convex and anterior part of the endplates should be removed as well to reinforce this effect. Screws are inserted in the center of the vertebral body parallel to the endplates and, according to the rotation of every single vertebra, parallel to the posterior wall. The first part of correction is obtained by setting the OR table back to a neutral position and traction of the patient. The rod is bent according to the intended sagittal contour; further correction is then achieved by cantilever technique, rotation of the prebent rod from scoliosis to kyphosis, and final compression. A structural graft or cage may be used at the lowest fused disc space to avoid a junctional thoracolumbar kyphosis (Figure 1).

\section{Lumbar curves (Lenke type 5)}

Correction and fusion are usually performed from end vertebra to end vertebra as well, typically T11 to L3. The approach is a thoracic-phrenic-lumbotomy with sectioning of the diaphragm close to the costal insertion. After resection of the discs and insertion of the screws, the prebent rod (bent according to the intended sagittal contour) is put in; correction is achieved by a cantilever technique when inserting the rod and by rotation of the prebent rod from scoliosis to lordosis. Structural spacers (e.g., cages) are inserted in the debrided disc spaces; compression is applied via the rod. Autologous bone or bone substitutes are added for bony fusion. 
Fixation can be performed with single or double rod instrumentation. Follow-up studies showed no significant difference in functional or radiographic outcomes between both types of instrumentation (10).

\section{Thoracoscopic surgery}

Video-assisted thoracoscopic surgery for AIS has been introduced in the 1990s. The goal was to achieve similar results like in open surgery with reduced approach morbidity: less muscle dissection, less postoperative pain, improved cosmesis, and less effect on the pulmonary function. The expectations were only partially answered. Compared to open thoracotomy, video-assisted thoracoscopic surgery had similar curve correction, a similar number of fused levels, and similar complication rates (11). Blood loss and operation theatre time seem to be equal or even higher (12).

\section{Postoperative treatment}

Patients are mobilized on the first postoperative day. A brace is not necessary. The patients are allowed to start with well-controlled sports like jogging, swimming, or bicycling three months after surgery. They may return to full activity six months after surgery.

\section{Results}

\section{Clinical and radiographic results}

Anterior instrumentation and fusion in AIS may deliver excellent results in short-term as well as long-term examinations.

In our series of 50 patients with thoracic and lumbar scoliosis, we evaluated clinical results with the SRS30 outcome score with a follow-up of 10 to 26 years (average 16 years): we found an average score of 4.1 (pain 4.2 / function 4.2/self-image 4.0/mental health 3.9/satisfaction 4.2); patients with thoracic scoliosis correction scored slightly better than patients with lumbar correction: 4.2 vs. 3.9 (13). These results are similar to a control group of volunteers without scoliosis (14).

Several recent publications endorse these favorable results. Tis et al. (15) described a series of 85 patients with thoracic curves (Lenke 1) in a five-year follow-up, Sudo et al. (16) a series of 25 patients with average 15 years follow-up, and Rushton et al. (17) 18 patients with 2 years follow-up. They found that anterior correction and fusion is a safe and reproducible procedure for thoracic AIS. The correction rate was between $51 \%$ and $64 \%$ in the coronal plane; the complication rate was low. The SRS outcome score improved significantly.

The advantages of anterior versus posterior techniques are the true segmental derotation with excellent rib hump correction (18). The rib hump showed better correction after anterior surgery than after posterior correction procedures (17).

Maintenance of thoracic curve correction after anterior instrumentation and fusion supplied a stable spontaneous correction of the second lumbar curve over time (19) (Figure 2).

For thoracolumbar/lumbar curves (Lenke 5), excellent results are described in short- and long-term followup as well. Verma et al. found an SRS score of average 4.4 two years after surgery (20), Kelly et al. a score of 4.3 and Sudo et al. of 4.2, both at 17 years follow-up $(21,22)$. The correction described in the coronal plane was between $74 \%$ and $80 \%$, with a powerful derotation of the apical vertebrae $(20,22,23)$. Complications like pseudoarthrosis and implant failures were rare $(10,23)$. High rates of satisfaction were described even in Lenke type 6 curves with partial reduction of the thoracic curve (24) (Figure 3).

\section{Sagittal profile}

Thoracic scoliosis is mostly associated with hypokyphosis or even lordosis in the sagittal plane. The three-dimensional degree of hypokyphosis is usually underestimated when plain lateral radiographs are used (25) (Figure 4).

Anterior correction and fusion can correct this hypokyphosis and restore a physiological sagittal profile. When comparing anterior and posterior approaches for correction of thoracic AIS, the results in frontal plane correction are similar; however, there is clear evidence that the anterior approach is superior in the restoration of a normal thoracic kyphosis (17,18,26-28).

These results are of special interest, as a thoracic flat back seems to be a risk factor for lumbar degenerative disc disease after spinal fusion in a long-term follow-up (29).

On the other hand, in patients with preoperative hyperkyphosis, a posterior approach should be considered. Furthermore, very young patients with high growth potential are at risk of developing hyperkyphosis during further growth after anterior correction and fusion.

\section{Fusion length}

In anterior scoliosis correction, the fusion is usually 

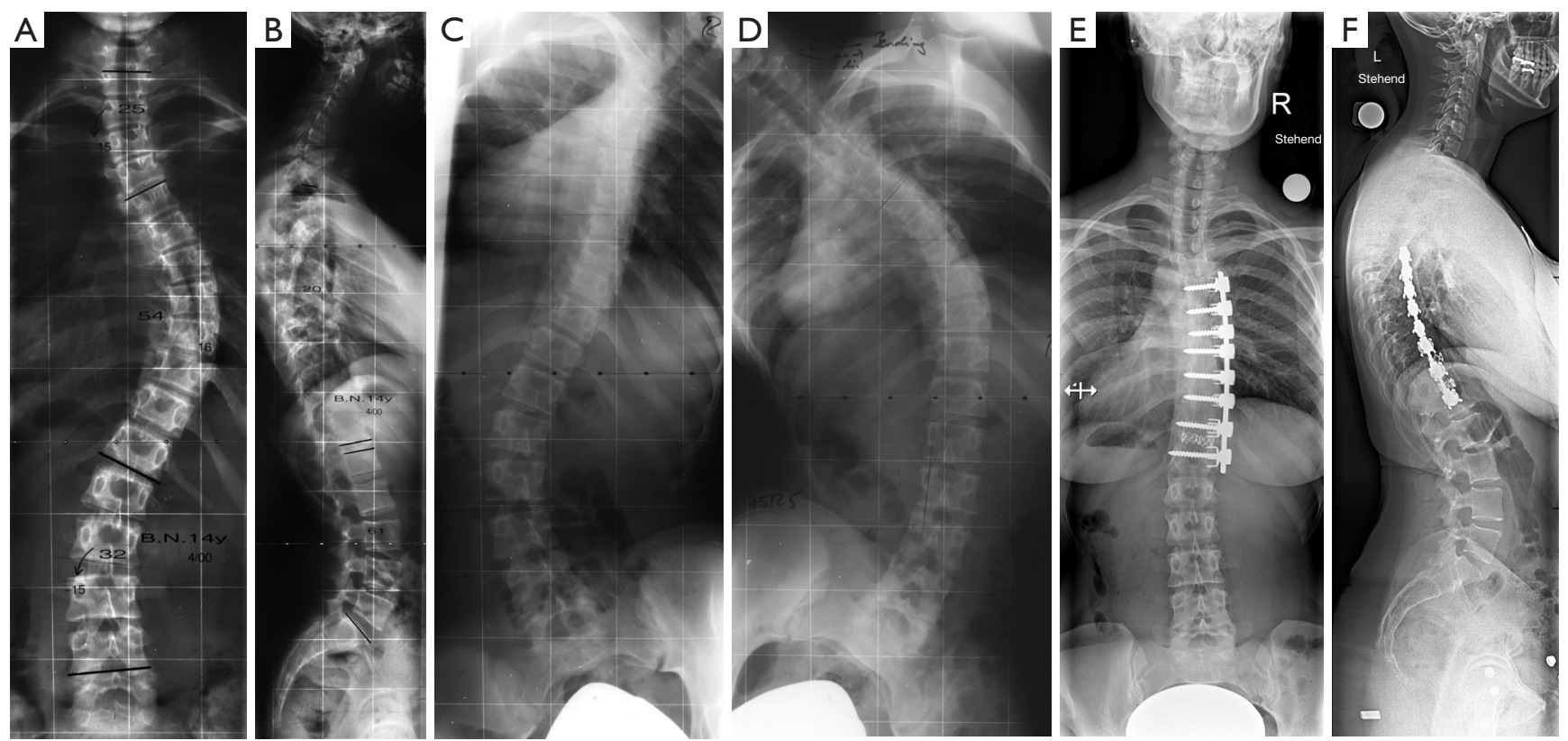

Figure 2 A 14 years old girl with adolescent idiopathic thoracic scoliosis. (A,B,C,D) Thoracic hypokyphosis causes cervical kyphosis; (E,F) the long-term follow-up radiographs 18 years after surgery shows the instrumentation T5-12 with solid fusion. The straight lumbar spine, no degenerative changes: sagittal profile is restored with physiological cervical lordosis.

performed from the upper-end vertebra to the lower end vertebra. Some authors recommend even shorter fusion segments down to three vertebrae/two segments in special cases to rebalance the spine (30). Current recommendations for posterior scoliosis correction comprise more segments in thoracic as well as in thoracolumbar/lumbar curves. The difference in fusion length is between one and fore vertebrae (26,28,31-36).

In our opinion, it is of utmost importance to keep the fusion length in these young patients as short as possible. This is especially valid for mobility in the lumbar segments. It may be accepted that the stress in a few residual mobile segments is higher and that this will result in an increased rate of degenerative disc disease during a lifetime (37).

\section{Pulmonary function}

After the anterior approach, due to the opening of the chest wall, the pulmonary function is more deteriorated than after a posterior approach. However, it improves within the next months. After two years, pulmonary function is comparable to the preoperative values without significant differences between anterior and posterior approaches $(38,39)$.

This was also found for thoracolumbar/lumbar AIS patients who underwent a thoracic-phrenic-laparotomy (40).

\section{Discussion}

The aim of surgery for AIS is a three-dimensional correction with a well-balanced, straight spine, and fusion as short as possible. A physiological sagittal profile has to be restored; the apical vertebrae have to be derotated. For a perfect cosmetic result, the shoulder level should be horizontalized, and the rib hump corrected.

Correction of thoracic deformity by an anterior approach is based on two mechanisms: First is the shortening of the anterior column of the spine, second the correction via the rigid rod. It has been shown that an aggressive anterior release with the removal of the rib heads, complete resection of the annulus, disruption of the posterior longitudinal ligament, and partial resection of the endplates of the adjacent vertebral bodies results in a considerable shortening of the anterior spine. Spontaneous correction of scoliosis follows this shortening without the application of any corrective force (41). In this case series with anterior release and posterior instrumentation, thoracic scoliosis corrected spontaneously from $80^{\circ}$ to $50^{\circ}$ after the anterior release, thoracic kyphosis improved from 

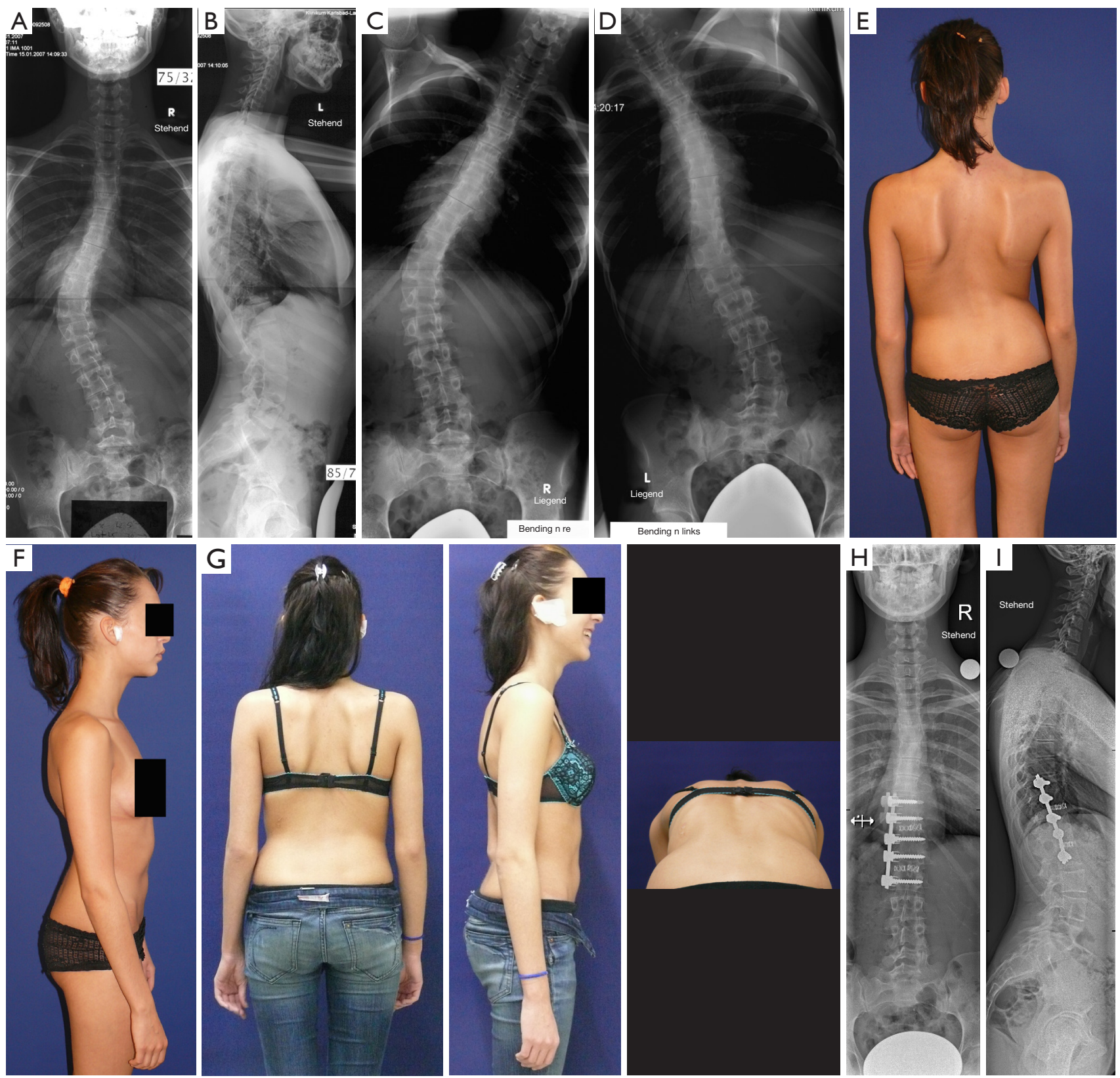

Figure 3 A 16 years old girl with a thoracolumbar scoliosis (Lenke 5C) (A,B,C,D,E,F), one year postoperatively following anterior correction and fusion $(\mathrm{G})$ and five years postoperatively: well-balanced spine with a short instrumentation and fusion (H,I).

$11^{\circ}$ to $32^{\circ}$. The reason for this effect is probably linked to the development of thoracic scoliosis: The imbalance of anterior and posterior spinal column length in favor of the anterior column may be a driving force in the development of thoracic AIS. The effect is a hypokyphotic and scoliotic spine. Anterior shortening reverses this imbalance; the spine straightens spontaneously, and kyphosis evolves (Figure 5).
The second part of the correction and the stabilization is then performed by the anterior instrumentation with a rigid rod by a cantilever procedure, rod rotation, and convex compression.

Surgery for AIS is to regard in the very long term. The main point is the quality of life of the patients within the following fifty or sixty years. Against this background, the 

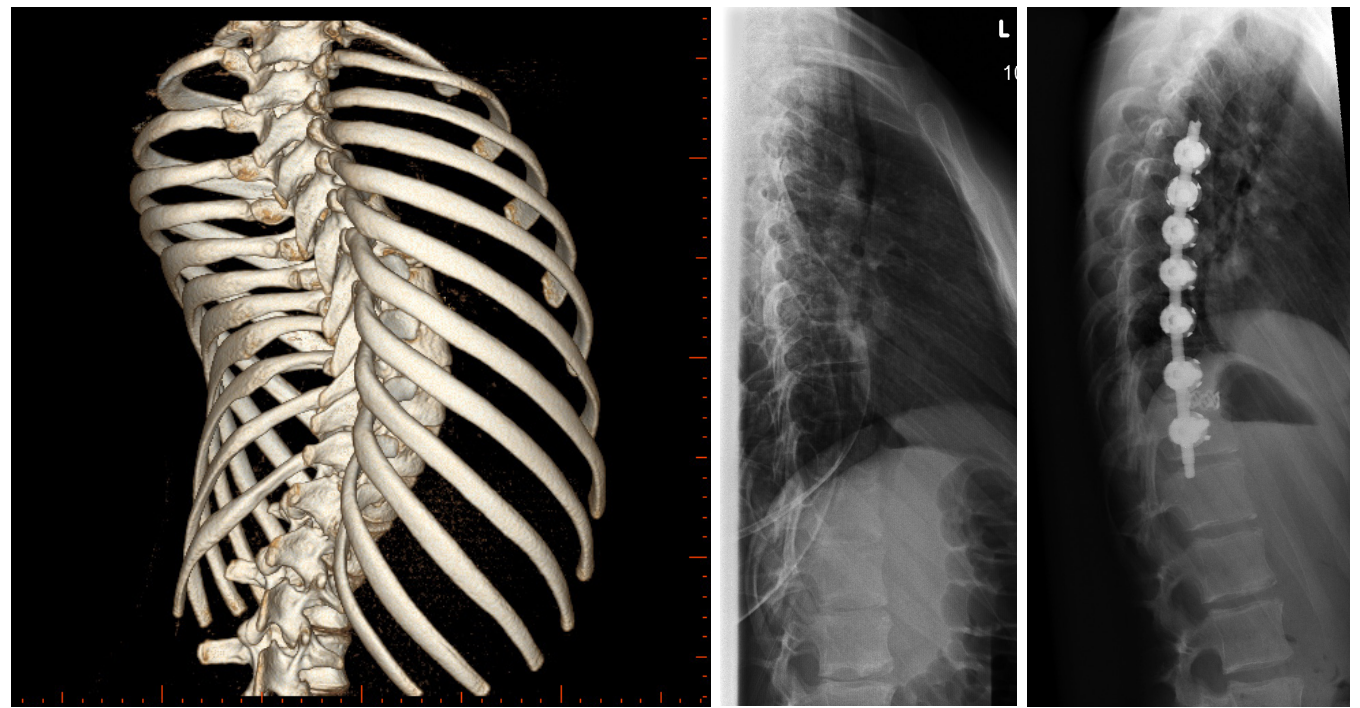

Figure 4 The 3D imaging reveals the severe hypokyphosis/lordosis in the scoliotic thoracic spine. Anterior shortening restores a physiologic sagittal profile.
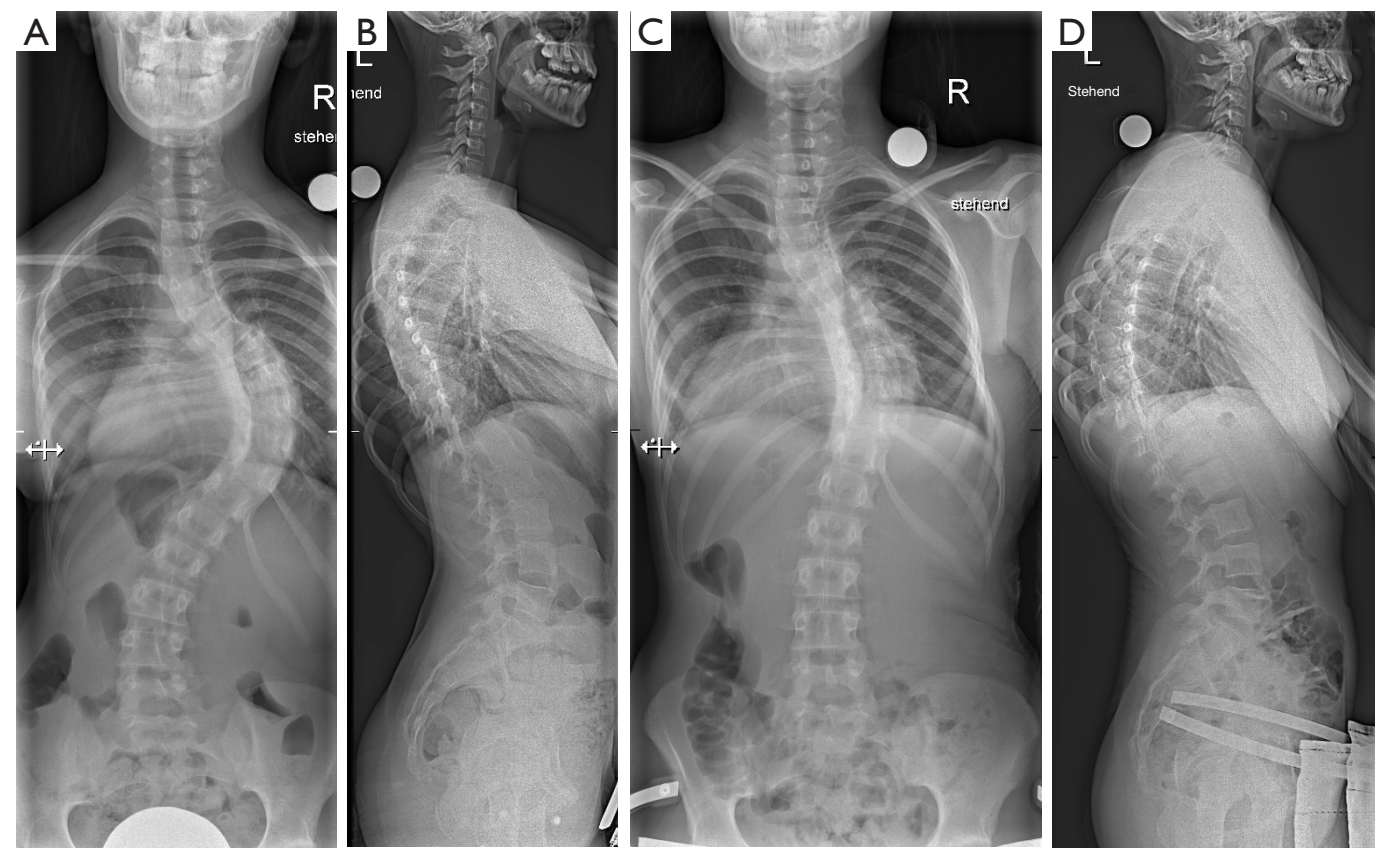

Figure 5 Rigid scoliosis Lenke 2A in a 12-year-old girl (A,B) and one week after anterior release with shortening of the anterior column of the spine without instrumentation (posterior instrumentation planned): spontaneous correction of scoliosis, restoration of thoracic kyphosis (standing position) (C,D).

approach for surgery is secondary. Time for surgery, size of the scares, and length of hospital stay may be secondary in the long run. It may even be secondary if there is a temporary decrease in pulmonary function. The authors believe that the most important goal of surgery has to be: to bring the spine into the best possible starting position for the next 50 years, to allow the patients a pain-free life with functional restrictions as less as possible. 
Factors that may have an impact on resilience and functionality of the spine in the long view and may reduce degeneration of the residual mobile spinal segments are:

(I) Fusion as short as possible to improve functionality, and to distribute the loads on as many mobile segments as possible to avoid early disc degeneration;

(II) Load of the remaining mobile segments in a physiological manner (less residual curve, physiological sagittal posture, horizontal upper and lower instrumented vertebra, derotation of the spine).

This has an impact on decision making for the approach in AIS surgery. An anterior or an anterior-posterior approach should be considered if thereby the fusion levels can be reduced, the sagittal profile can be improved, or a better derotation can be achieved. These advantages will persist for a lifetime; most of the disadvantages are only temporarily.

\section{Acknowledgments}

None.

\section{Footnote}

Conflicts of Interest: The authors have no conflicts of interest to declare.

Ethical Statement: The authors are accountable for all aspects of the work in ensuring that questions related to the accuracy or integrity of any part of the work are appropriately investigated and resolved.

\section{References}

1. Dwyer AF, Schafer MF. Anterior approach to scoliosis. Results of treatment in fifty-one cases. J Bone Joint Surg Br 1974;56:218-24.

2. Hsu LC, Zucherman J, Tang SC, et al. Dwyer instrumentation in the treatment of adolescent idiopathic scoliosis. J Bone Joint Surg Br 1982;64:536-41.

3. Hammerberg KW, Zielke, K. VDS instrumentation for idiopathic thoracic curvatures. Presented at the Annual Meeting of the American Academy of Orthopedic Surgeons, Las Vegas. 1985.

4. Harms J, Jeszenszky D, Beele B. Ventral correction of thoracic scoliosis. In Bridwell KH, DeWald RD. editors.
The Textbook of Spinal Surgery 2nd ed. Philadelphia: Lippincott-Raven, 1997:611-26.

5. Kaneda K, Shono Y, Satoh S, et al. Anterior correction of thoracic scoliosis with Kaneda anterior spinal system. A preliminary report. Spine 1997;22:1358-68.

6. Halm HF, Liljenquist U, Niemeyer T, et al. Halm-Zielke instrumentation for primary stable anterior scoliosis surgery: operative technique and 2-year results in ten consecutive adolescent idiopathic scoliosis patients within a prospective clinical trial. Eur Spine J 1998;7:429-34.

7. Betz RR, Harms J, Clements DH 3rd, et al. Comparison of anterior and posterior instrumentation for correction of adolescent thoracic idiopathic scoliosis. Spine 1999;24:225-39.

8. Lonner BS, Ren Y, Yaszay B, et al. Evolution of Surgery for Adolescent Idiopathic Scoliosis Over 20 Years: Have Outcomes Improved? Spine 2018;43:402-10.

9. Lenke LG, Betz RR, Harms J, et al. Adolescent idiopathic scoliosis: a new classification to determine extent of spinal arthrodesis. J Bone Joint Surg Am 2001;83:1169-81.

10. Nambiar M, Yang Y, Liew S, et al. Single- versus dual-rod anterior instrumentation of thoracolumbar curves in adolescent idiopathic scoliosis. Eur Spine J 2016;25:3249-55.

11. Padhye K, Soroceanu A, Russell D, et al. Thoracoscopic Anterior Instrumentation and Fusion as a Treatment for Adolescent Idiopathic Scoliosis: A Systematic Review of the Literature. Spine Deform 2018;6:384-90.

12. Grewal H, Betz RR, D'Andrea LP, et al. A prospective comparison of thoracoscopic vs open anterior instrumentation and spinal fusion for idiopathic thoracic scoliosis in children. J Pediatr Surg 2005;40:153-6; discussion 156-7.

13. Ruf M, Letko L, Ostrowski G, et al. Langzeitergebnisse nach ventraler Korrektur der idiopathischen

Adoleszentenskoliose. In: Süddeutscher Orthopädenkongress. Baden-Baden: VSOU, 2014.

14. Akazawa T, Minami S, Kotani T, et al. Health-related quality of life and low back pain of patients surgically treated for scoliosis after 21 years or more of follow-up: comparison among nonidiopathic scoliosis, idiopathic scoliosis, and healthy subjects. Spine 2012;37:1899-903.

15. Tis JE, O'Brien MF, Newton PO, et al. Adolescent idiopathic scoliosis treated with open instrumented anterior spinal fusion: five-year follow-up. Spine 2010;35:64-70.

16. Sudo H, Ito M, Kaneda K, et al. Long-term outcomes of anterior spinal fusion for treating thoracic adolescent 
idiopathic scoliosis curves: average 15-year follow-up analysis. Spine 2013;38:819-26.

17. Rushton PR, Grevitt MP, Sell PJ. Anterior or posterior surgery for right thoracic adolescent idiopathic scoliosis (AIS)? A prospective cohorts' comparison using radiologic and functional outcomes. J Spinal Disord Tech 2015;28:80-8.

18. Liljenqvist U, Halm H, Bullmann V. Spontaneous lumbar curve correction in selective anterior instrumentation and fusion of idiopathic thoracic scoliosis of Lenke type C. Eur Spine J 2013;22 Suppl 2:S138-48.

19. Sudo HS, Mayer MM, Kaneda KK, et al. Maintenance of spontaneous lumbar curve correction following thoracic fusion of main thoracic curves in adolescent idiopathic scoliosis. Bone Joint J 2016;98:997-1002.

20. Verma K, Auerbach JD, Kean KE, et al. Anterior spinal fusion for thoracolumbar scoliosis: comprehensive assessment of radiographic, clinical, and pulmonary outcomes on 2-years follow-up. J Pediatr Orthop 2010;30:664-9.

21. Kelly DM, McCarthy RE, McCullough FL, et al. Long-term outcomes of anterior spinal fusion with instrumentation for thoracolumbar and lumbar curves in adolescent idiopathic scoliosis. Spine 2010;35:194-8.

22. Sudo H, Ito M, Kaneda K, et al. Long-term outcomes of anterior dual-rod instrumentation for thoracolumbar and lumbar curves in adolescent idiopathic scoliosis: a twelve to twenty-three-year follow-up study. J Bone Joint Surg Am 2013;95:e49.

23. Delfino R, Pizones J, Ruiz-Juretschke C, et al. Selective Anterior Thoracolumbar Fusion in Adolescent Idiopathic Scoliosis: Long-Term Results After 17-Year Follow-Up. Spine 2017;42:E788-94.

24. Direito-Santos B, Queirós CM, Serrano P, et al. Long-Term Follow-Up of Anterior Spinal Fusion for Thoracolumbar/Lumbar Curves in Adolescent Idiopathic Scoliosis. Spine 2019;44:1137-43.

25. Parvaresh KC, Osborn EJ, Reighard FG, et al. Predicting 3D Thoracic Kyphosis Using Traditional 2D Radiographic Measurements in Adolescent Idiopathic Scoliosis. Spine Deform 2017;5:159-65.

26. Lowe TG, Betz R, Lenke L, et al. Anterior single-rod instrumentation of the thoracic and lumbar spine: saving levels. Spine 2003;28:S208-16.

27. Chaloupka R, Repko M, Tichý V, et al. [Comparison of two surgical methods for treatment of idiopathic thoracic scoliosis - anterior versus posterior approaches]. Acta Chir Orthop Traumatol Cech 2012;79:422-8.
28. Newton PO, Marks MC, Bastrom TP, et al. Harms Study Group. Surgical treatment of Lenke 1 main thoracic idiopathic scoliosis: results of a prospective, multicenter study. Spine 2013;38:328-38.

29. Bernstein P, Hentschel S, Platzek I, et al. Thoracal flat back is a risk factor for lumbar disc degeneration after scoliosis surgery. Spine J 2014;14:925-32.

30. Hall JE, Millis MB, Snyder BD. Short Segment Anterior Instrumentation for Thoracolumbar Scoliosis. In: Bridwell KH, DeWald RL. editors. The Textbook of Spinal Surgery. 2nd ed. Philadelphia: Lippincott-Raven, 1997:665-74.

31. Tao F, Wang Z, Li M, et al. A comparison of anterior and posterior instrumentation for restoring and retaining sagittal balance in patients with idiopathic adolescent scoliosis. J Spinal Disord Tech 2012;25:303-8.

32. Luo M, Wang W, Shen M, et al. Anterior versus posterior approach in Lenke 5C adolescent idiopathic scoliosis: a meta-analysis of fusion segments and radiological outcomes. J Orthop Surg Res 2016;11:77.

33. Miyanji F, Nasto LA, Bastrom T, et al. A Detailed Comparative Analysis of Anterior Versus Posterior Approach to Lenke 5C Curves. Spine 2018;43:E285-91.

34. O'Donnell C, Michael N, Pan X, et al. Anterior Spinal Fusion and Posterior Spinal Fusion Both Effectively Treat Lenke Type 5 Curves in Adolescent Idiopathic Scoliosis: A Multicenter Study. Spine Deform 2018;6:231-40.

35. Vavruch L, Brink RC, Malmqvist M, et al. Surgical Outcomes of Anterior Versus Posterior Fusion in Lenke Type 1 Adolescent Idiopathic Scoliosis. Spine 2019;44:E823-32.

36. Yoshihara H. Surgical Treatment of Lenke Type 5 Adolescent Idiopathic Scoliosis: A Systematic Review. Spine 2019;44:E788-99.

37. Lonner BS, Ren Y, Upasani VV, et al. Disc Degeneration in Unfused Caudal Motion Segments Ten Years Following Surgery for Adolescent Idiopathic Scoliosis. Spine Deform 2018;6:684-90.

38. Bullmann V, Schulte TL, Schmidt C, et al. Pulmonary function after anterior double thoracotomy approach versus posterior surgery with costectomies in idiopathic thoracic scoliosis. Eur Spine J 2013;22:S164-71.

39. Lee AC, Feger MA, Singla A, et al. Effect of Surgical Approach on Pulmonary Function in Adolescent Idiopathic Scoliosis Patients: A Systemic Review and Meta-analysis. Spine 2016;41:E1343-55.

40. Ruiz-Juretschke C, Pizones J, Delfino R, et al. Long-term 
Pulmonary Function After Open Anterior Thoracolumbar Surgery in Thoracolumbar/Lumbar Idiopathic Adolescent Scoliosis. Spine 2017;42:1241-7.

Cite this article as: Ruf M, Drumm J, Jeszenszky D. Anterior instrumented fusion for adolescent idiopathic scoliosis. Ann Transl Med 2020;8(2):31. doi: 10.21037/atm.2019.11.84
41. Ruf M, Letko L, Matis N, et al. The Effect of Anterior Mobilisation and Shortening in the Correction of Rigid Idiopathic Thoracic Scoliosis. Spine 2013;38:E1662-8. 